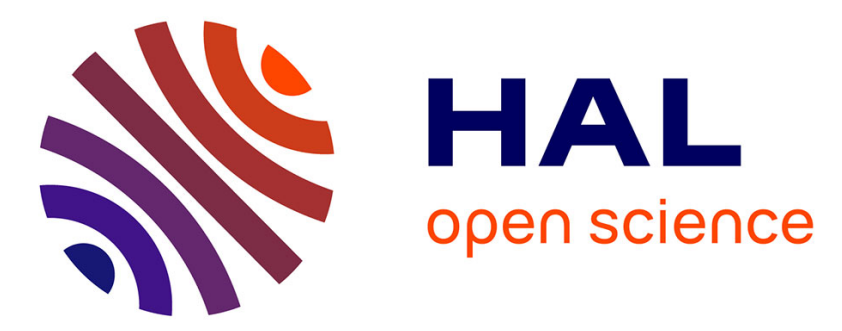

\title{
Temperature dependent locally resolved 13C Knight shifts in the organic conductor $\mathrm{TTF}[\mathrm{Ni}(\mathrm{dmit}) 2] 2$
}

A. Vainrub, E. Canadell, D. Jérome, P. Bernier, T. Nunes, M.-F. Bruniquel, P. Cassoux

\section{- To cite this version:}

A. Vainrub, E. Canadell, D. Jérome, P. Bernier, T. Nunes, et al.. Temperature dependent locally resolved 13C Knight shifts in the organic conductor TTF[Ni(dmit)2]2. Journal de Physique, 1990, 51 (21), pp.2465-2476. 10.1051/jphys:0199000510210246500 . jpa-00212544

\section{HAL Id: jpa-00212544 https://hal.science/jpa-00212544}

Submitted on 1 Jan 1990

HAL is a multi-disciplinary open access archive for the deposit and dissemination of scientific research documents, whether they are published or not. The documents may come from teaching and research institutions in France or abroad, or from public or private research centers.
L'archive ouverte pluridisciplinaire HAL, est destinée au dépôt et à la diffusion de documents scientifiques de niveau recherche, publiés ou non, émanant des établissements d'enseignement et de recherche français ou étrangers, des laboratoires publics ou privés. 
Classification

Physics Abstracts

74.70K $-71.45 \mathrm{~L}$

\title{
Temperature dependent locally resolved ${ }^{13} \mathrm{C}$ Knight shifts in the organic conductor TTF $\left[\mathrm{Ni}(\text { dmit })_{2}\right]_{2}$
}

\author{
A. Vainrub $\left({ }^{1, *}\right)$, E. Canadell $\left({ }^{2}\right)$, D. Jérome $\left({ }^{1}\right)$, P. Bernier $\left({ }^{3}\right)$, T. Nunes $\left({ }^{3}\right)$, \\ M.-F. Bruniquel $\left({ }^{4}\right)$ and P. Cassoux $\left({ }^{4}\right)$ \\ (') Laboratoire de Physique des Solides, Université de Paris-Sud, 91405 Orsay, France \\ (2) Laboratoire de Chimie Théorique, Université de Paris-Sud, 91405 Orsay, France \\ $\left({ }^{3}\right)$ Groupe de Dynamique des Phases Condensées, Université des Sciences et Techniques du \\ Languedoc, 34060 Montpellier, France \\ $\left({ }^{4}\right)$ Laboratoire de Chimie de Coordination du CNRS, Université Paul Sabatier, 31077 Toulouse, \\ France
}

(Received 18 May 1990, accepted in final form 18 July 1990)

\begin{abstract}
High resolution ${ }^{13} \mathrm{C}$ NMR results for the organic conductor TTF[Ni(dmit) $]_{2}$ enriched by ${ }^{13} \mathrm{C}$ isotope in $\mathrm{Ni}(\mathrm{dmit})_{2}$ molecules are reported. Variable temperature magic angle spinning experiments were performed between 160 and $295 \mathrm{~K}$. Paramagnetic Knight shifts in the range 41-106.5 ppm at room temperature are observed for all $\mathrm{Ni}(\mathrm{dmit})_{2}$ carbon sites. These shifts are slightly temperature dependent for inner carbons. However, for outer carbons, the shifts strongly decrease towards low temperature due to precursor effects of the charge density wave (CDW) transition. The results confirm the occurrence of both HOMO (highest occupied molecular orbital) and LUMO (lowest unoccupied molecular orbital) conduction bands and are compared with calculated partial contributions of individual carbon atoms to the density of states at the Fermi level. We suggest that CDW effects above $160 \mathrm{~K}$ are associated with the LUMO bands.
\end{abstract}

\section{Introduction.}

Organic conductors based on transition metal $(\mathrm{M}=\mathrm{Ni}, \mathrm{Pd}, \mathrm{Pt})$ complexes of dmit (dmit = 1,3-dithia-2-thione-4,5-dithiolato) [1] have attracted a great deal of attention since the discovery of superconductivity in $\mathrm{TTF}\left[\mathrm{Ni}(\mathrm{dmit})_{2}\right]_{2}(\mathrm{TTF}=$ tetrathiafulvalene) at $1.6 \mathrm{~K}$ under a pressure of $7 \mathrm{kbar}$ [2]. Two other superconducting compounds of this family, $\left(\mathrm{CH}_{3}\right)_{4} \mathrm{~N}\left[\mathrm{Ni}(\mathrm{dmit})^{2}\right]^{2} \quad\left(T_{\mathrm{c}}=5 \mathrm{~K}\right.$ at $\left.7 \mathrm{kbar}\right)[3]$ and $\alpha^{\prime}-\mathrm{TTF}\left[\mathrm{Pd}(\mathrm{dmit})_{2}\right]_{2} \quad\left(T_{\mathrm{c}}=6.5 \mathrm{~K}\right.$ at $20 \mathrm{kbar})$ [4] were found later. Moreover, an interesting feature of $\mathrm{TTF}\left[\mathrm{M}(\mathrm{dmit})_{2}\right]_{2}(\mathrm{M}=\mathrm{Ni}$, Pd) is the occurrence of charge density wave (CDW) instabilities observed in X-ray diffuse scattering studies at ambient pressure [5]. Thus, these materials provide an interesting example of competition between CDW and superconductivity in molecular metals.

(*) Permanent address : Institute of Chemical Physics and Biophysics, Estonian Academy of Sciences, 200001 Tallinn, Estonia, U.S.S.R. 
In contrast with other organic conductors, the CDW coexists with metal like conductivity in TTF $\left[\mathrm{Ni}(\mathrm{dmit})_{2}\right]_{2}$. Proton spin-lattice relaxation studies of the TTF stacks show that their metallic character is retained down to $1 \mathrm{~K}$ with no indication of CDW effects [6]. We have recently reported ${ }^{13} \mathrm{C}$ NMR experiments on samples enriched by ${ }^{13} \mathrm{C}$ isotope in $\mathrm{Ni}(\mathrm{dmit})_{2}$ molecules only [7]. These measurements give evidence that the CDW involves the Ni(dmit) ${ }_{2}$ stacks. The Knight shift decreases but not vanishes at low temperature showing that the $\mathrm{Ni}(\mathrm{dmit})_{2}$ subsystem is metallic in the entire temperature domain. Two possible explanations for why the CDW does not induce a metal-insulator transition on the $\mathrm{Ni}(\mathrm{dmit})_{2}$ stacks were suggested [7]. The first assumed imperfect nesting of a wraped planar 1D Fermi surface. The second was based on the prediction of a multiconduction band for the $\mathrm{Ni}(\mathrm{dmit})_{2}$ stacks consisting of the HOMO and LUMO bands [8]. The CDW would open a gap in only some of them so that some metallic bands will remain. We were unable to distinguish between these two possibilities from broad band NMR data. As it will be seen, the results of the present work provide support for the second explanation.

We report here high resolution variable temperature ${ }^{13} \mathrm{C}$ NMR measurements on TTF[Ni(dmit $\left.)_{2}\right]_{2}$ enriched by ${ }^{13} \mathrm{C}$ isotope in $\mathrm{Ni}(\mathrm{dmit})_{2}$ molecules. The paper is organized as follows. In section 2 we present experimental results, analyze the assignments of the resonance lines and the local Knight shifts for different carbon sites are determined. A brief account of the band electronic structure of the $\mathrm{Ni}(\mathrm{dmit})_{2}$ slabs in TTF[Ni(dmit $\left.)_{2}\right]_{2}$ and the calculated densities of states at the Fermi level are presented in section 3 . These results are used in section 4 to roughly estimate the contributions of the HOMO and LUMO bands to the Knight shifts and to inquire about the origin of the CDW. Finally, section 5 contains a summary of the results and some suggestions for further experiments.

\section{High resolution NMR spectra. Assignment of the lines.}

The experiments were carried out on a microcrystalline powder sample $(40 \mathrm{mg})$ of TTF $\left[\mathrm{Ni}(\mathrm{dmit})_{2}\right]_{2}$ nonselectively enriched to $50 \%{ }^{13} \mathrm{C}$ isotope content on $\mathrm{Ni}(\mathrm{dmit})_{2}$ molecules. The preparation procedure has been already reported [7]. ${ }^{13} \mathrm{C}$ magic angle spinning (MAS) spectra were measured at $50.3 \mathrm{MHz}$ (Bruker CXP-200 or MSL-200 high power spectrometers) with or without cross polarization (CP) using variable temperature $(160-300 \mathrm{~K}) \mathrm{MAS}$ probehead with cylindrical rotor spinning up to $4.6 \mathrm{kHz}$ rotation rate. Some experiments were performed at $90.5 \mathrm{MHz}$ on a Bruker MSL-360 spectrometer. All the NMR shifts were measured with tetramethyl-silane (TMS) scale as the reference.

Figure 1 shows examples of ${ }^{13} \mathrm{C}$ MAS NMR spectra at room temperature and $185 \mathrm{~K}$. These spectra were obtained without polarization transfer since some resonances, as we discuss below, are very weak in cross polarization experiments. Six isotropic lines are observed and denoted as A, B, C, D, E and F. All other signals are well known spinning side bands (SSB) which give for each isotropic line a pattern of uniformly separated lines with the spacing equal to a frequency of the sample rotation. As usual, we have distinguished between isotropic lines and SSB's by change of the rotation rate. Figure 1 illustrates the temperature behaviour of different lines. The overall temperature dependence of the resonance positions is shown in figure 2. The A and B lines shift upfield whereas the D and E ones shift less downfield with the temperature decrease. The positions of the $\mathrm{C}$ and $\mathrm{F}$ lines are almost temperature independent.

The spectrum in the extended range and the assignments of the SSB's are shown in figure 3. Several SSB's are observed within a range of about $350 \mathrm{ppm}$ for each of the C, D, E and F lines, but the A and B lines have no side brands. The SSB pattern is known to be directly related to the anisotropy of the magnetic shielding tensor [9]. The side bands are strong within a range of the anisotropic magnetic shifts. The spectrum in figure 3 was obtained at $50.3 \mathrm{MHz}$ 


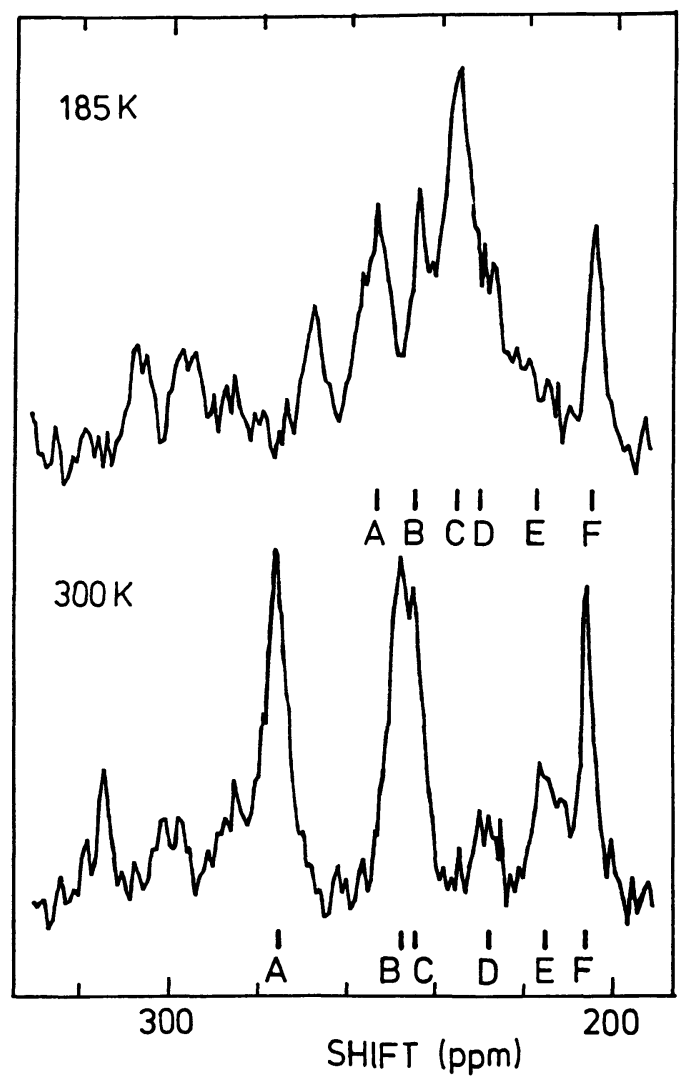

Fig. 1. - ${ }^{13} \mathrm{C}$ MAS NMR spectra of TTF[Ni(dmit) $]_{2}$ measured without cross polarization at $185 \mathrm{~K}$. A, $\mathrm{B}, \mathrm{C}, \mathrm{D}, \mathrm{E}$ and $\mathrm{F}$ denote isotropic lines. All other signals signals are spinning side bands.

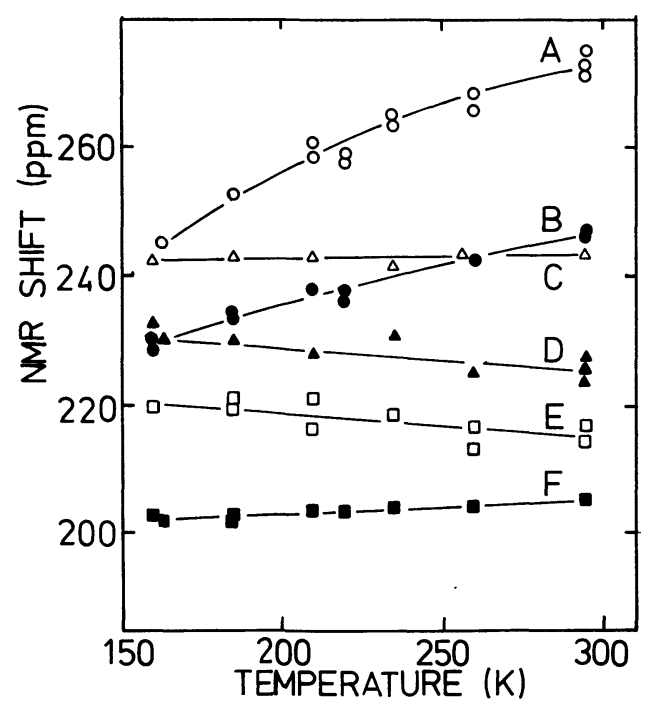

Fig. 2. - Positions of ${ }^{13} \mathrm{C}$ MAS NMR resonances of TTF[Ni(dmit) $]_{2}$ vs. temperature. 


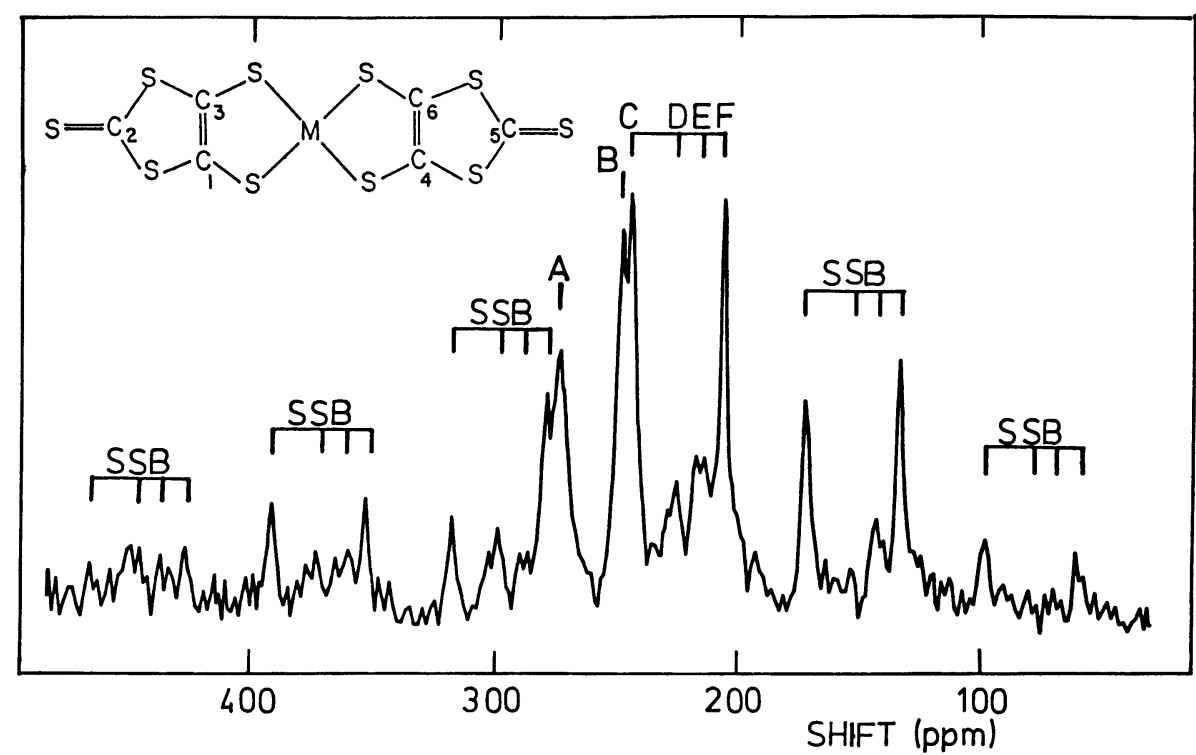

Fig. 3. - $50.3 \mathrm{MHz}{ }^{13} \mathrm{C}$ MAS NMR spectrum of TTF[Ni(dmit $\left.)_{2}\right]_{2}$. The identification of isotropic lines and their spinning side bands (SSB) is shown. Spinning rate is $3690 \mathrm{~Hz}$. Shown in the insert is the $\mathrm{Ni}(\mathrm{dmit})_{2}$ formula with the numbering for different carbon sites.

with MAS speed $\nu_{\mathrm{r}}=3690 \mathrm{~Hz}=73.4 \mathrm{ppm}$. So we estimate $150 \mathrm{ppm}$ as an upper limit for the anisotropy of the magnetic shift tensor for the $A$ and $B$ lines and $350 \mathrm{ppm}$ as a lower limit for that of the $\mathrm{C}, \mathrm{D}, \mathrm{E}$ and $\mathrm{F}$ lines.

We would like to consider now the assignment of the lines to different carbon sites of $\mathrm{Ni}(\mathrm{dmit})_{2}$ stacks. The four $\mathrm{Ni}(\mathrm{dmit})_{2}$ molecules in the monoclinic unit cell of TTF $\left[\mathrm{Ni}(\mathrm{dmit})_{2}\right]_{2}$ are crystallographically equivalent [1]. But the six carbon sites in a given $\mathrm{Ni}(\mathrm{dmit})_{2}$ molecule are nonequivalent in the crystal (see insert in Fig. 3 for the Ni(dmit) formula and numbering of carbons). We summarize NMR data in table I where the results of our spin-lattice relaxation measurements are also included. The relaxation of $\mathbf{A}$ and $\mathbf{B}$ lines is 3-4 times slower than those for other lines. It is clear from table I that on the basis of similarity

Table I. - Characteristics of the ${ }^{13} \mathrm{C}$ MAS NMR spectrum of $\mathrm{TTF}\left(\mathrm{Ni}(\mathrm{dmit})_{2}\right)_{2}$. For each resonance line are given : possible assignments, the shift at room temperature $(\delta$, in $\mathrm{ppm})$ and its temperature dependence $(\mathrm{d} \delta / \mathrm{d} T$, in $\mathrm{ppm} / \mathrm{K})$, an estimate of the shift's anisotropy $\left(\delta_{\text {aniso }}\right.$ in $\left.\mathrm{ppm}\right)$, the spin-lattice relaxation rate $\left(1 / T_{1}\right.$, in $\left.\mathrm{s}^{-1}\right)$ and the Knight shift $(K$, in $\mathrm{ppm})$.

\begin{tabular}{|c|c|c|c|c|c|c|}
\hline Resonance & Nucleus & $\delta$ & $\mathrm{d} \delta / \mathrm{d} T$ & $\boldsymbol{\delta}_{\text {aniso }}$ & $1 / T_{1}$ & $K$ \\
\hline A & C2(C5) & 273.5 & 0.19 & $<150$ & 3.2 & 68 \\
\hline B & $\mathrm{C5}(\mathrm{C} 2)$ & 246.5 & 0.12 & $<150$ & 2.6 & 41 \\
\hline A & C1, C3) & 243.5 & 0.005 & $\geqslant 350$ & 9 & 106.5 \\
\hline $\mathrm{D}$ & $(\mathrm{C} 4, \mathrm{C} 6)$ & 225.5 & -0.038 & $\geqslant 350$ & 11 & 88 \\
\hline E & C4, C6 & 215 & -0.044 & $\geqslant 350$ & 11 & 78 \\
\hline $\mathrm{F}$ & $(\mathrm{C} 1, \mathrm{C} 3)$ & 205 & 0.02 & $\geqslant 350$ & 9 & 68 \\
\hline
\end{tabular}


of behaviour all lines can be divided into two groups. The first one consists of $\mathrm{A}$ and $\mathrm{B}$ lines characterized by a strong temperature dependence of the position, small anisotropy of the magnetic shift and slow spin-lattice relaxation. C, D, E and F lines with weak temperature dependence, stronger anisotropy and faster relaxation form the second group. Since similar NMR properties of alike sites on the $\mathrm{Ni}(\mathrm{dmit})_{2}$ unit can be expected, we propose that $\mathrm{A}$ and $B$ can be associated with the outer sites $\mathrm{C} 2$ and $\mathrm{C} 5$ and $\mathrm{C}, \mathrm{D}, \mathrm{E}$ and $\mathrm{F}$ with the inner sites $\mathrm{C1}$, C3, C4 and C6. This analysis corroborates the inhomogeneity of relaxation rate which was noticed in the wide-band spectra [7]. The absence of any Korringa-like relationship between values of $T_{1}^{-1}$ and $K$ (namely $1 / T_{1} \alpha K^{2}$ ) in table I could possibly be ascribed to the existence of an enhancement factor for the relaxation due to correlations which is not uniform over the dmit molecule.

The cross polarization experiments provide further evidence for these assignments. Figure 4 shows spectra with the same number of accumulations for different polarization transfer contact times at room temperature. By comparison of these results with those without CP shown in figures 1 and 3 two observations can be made. First, there is a strong enhancement of the A and B lines with respect to the other ones. Second, the intensities of the $A$ and $B$ lines grow faster and saturate earlier with a contact time increase. Thus the cross polarization is more effective and faster for the A and B lines. This is in accordance with their assignment to outer carbons since the distances from TTF protons to outer carbons (the shortest contact is $4.2 \AA$ ) are shorter than those to inner carbons (about $6.1 \AA$ ).

Since our assignments are incomplete, we have tried to get additional information from rotational resonance experiments [10]. The effect is known for isolated homonuclear dipolar-

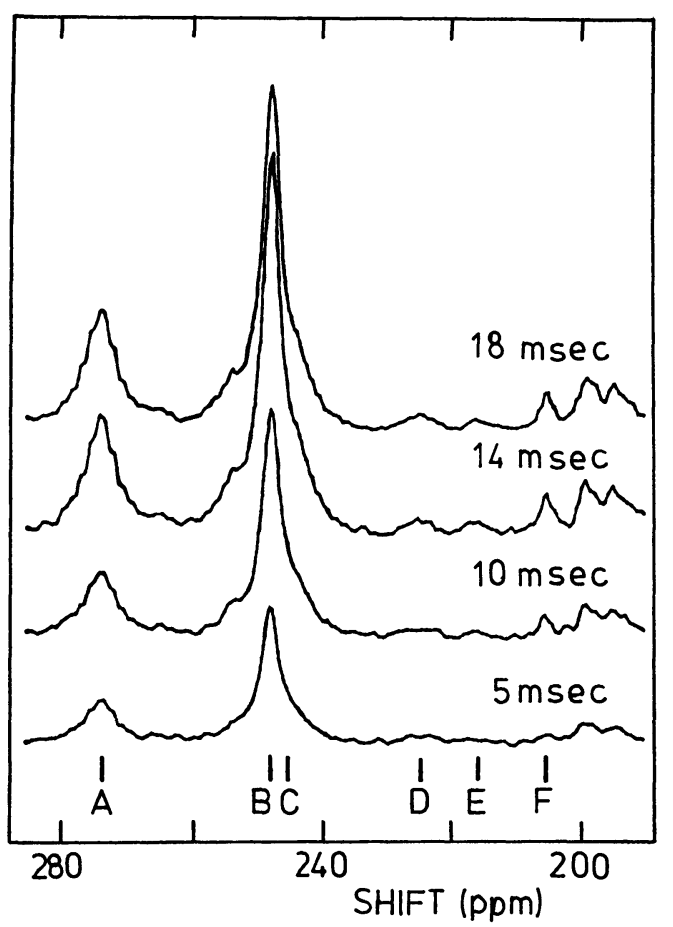

Fig. 4. - ${ }^{13} \mathrm{C}$ MAS cross polarization NMR spectra of TTF[Ni(dmit $\left.)_{2}\right]_{2}$ for different polarization transfer contact times. The, number of accumulations (1 866 scans) is the same for each spectrum. 
coupled spin pairs. The normally sharp resonance lines broaden and split if the frequency of magic angle spinning $\nu_{\mathrm{r}}$ is adjusted to satisfy the condition $\Delta \omega_{\text {iso }}=2 \pi \nu_{\mathrm{r}} n$. Here, $\Delta \omega_{\text {iso }}$ is the difference in isotropic magnetic shifts and $\mathrm{n}$ is an integer. The dipolar coupling of two inner ${ }^{13} \mathrm{C}$ spins of the same dmit moiety is stronger than their other homonuclear dipolar interactions. Consequently, $\mathrm{C} 1-\mathrm{C} 3$ and $\mathrm{C} 4-\mathrm{C} 6$ are fairly isolated spin pairs. The spinning rate of about $1700 \mathrm{~Hz}$ is sufficient to obtain narrow lines for TTF[Ni(dmit) $\left.{ }_{2}\right]_{2}$. Using 50.3 or $90.5 \mathrm{MHz}{ }^{13} \mathrm{C}$ MAS NMR we were able to adjust resonance rotation speeds for all pairs of lines with separation larger than $19 \mathrm{ppm}$. However we did not succeed to find spin pairs : in all cases, no changes of line shapes were observed. So we believe the differences of the shifts within C1-C3 and C4-C6 pairs do not exceed 19 ppm. These experiments have shown that CE, C-F and D-F pairs of inner carbon lines do not belong to spin pairs. Therefore the only possibility which remains is to associate $\mathrm{C}-\mathrm{D}$ and E-F pairs of lines with spin pairs $\mathrm{C} 1-\mathrm{C} 3$ and C4-C6. Unfortunately, we could not perform rotational resonance for C-D and E-F line pairs because of the small shift differences. It was possible however to study the most separated A line together with each other line and so its assignement to an outer site was confirmed. The final results of our assignments are shown in table I.

The total magnetic shift for an organic conductor is a sum of the chemical shift and the Knight shift. We have measured the chemical shifts to be $205.6 \mathrm{ppm}$ for outer sites and $137 \mathrm{ppm}$ (really a doublet 136.5 and $137.5 \mathrm{ppm}$ is observed) for inner carbon sites in nonconducting $\left(\mathrm{NBu}_{4}\right)_{2}\left[\mathrm{Zn}(\mathrm{dmit})_{2}\right]$. Using these values we have determined the locally resolved Knight shifts of 41-106.5 ppm as shown in table $I$.

\section{Electronic band structure calculations.}

In order to gain some insight into the origin of the temperature dependence of the ${ }^{13} \mathrm{C}$ MAS NMR resonances we carried out tight-binding band structure calculations on the $\mathrm{Ni}(\mathrm{dmit})_{2}$ slabs of TTF[Ni(dmit $\left.)_{2}\right]_{2}$. An effective one-electron hamiltonian of the extended Hückel type [11] was used. All valence electrons were explicitely taken into account in the calculations. The basis set consisted of Slater type orbitals of double- $\zeta$ quality for nickel $3 \mathrm{~d}$ functions and of single- $\zeta$ quality for carbon $2 s$ and $2 p$, sulfur $3 s$ and $3 p$ and nickel $4 s$ and $4 p$ [12]. The exponents, contraction coefficients and atomic parameters used in the calculations are summarised in table II. As usual in organometallic compounds, the off-diagonal matrix elements of the hamiltonian were calculated according to the modified Wolfsberg-Helmholz formula [13]. The calculations were performed on the room temperature and ambient pressure structure [1] which is the only presently available.

Table II. - Exponents and parameters used in the calculations.

\begin{tabular}{|l|c|c|c|c|c|c|}
\hline \multicolumn{1}{|c|}{ atom } & orbital & $H_{\mu \mu}(\mathrm{eV})$ & $\zeta_{1}$ & $\zeta_{2}$ & $c_{1}^{(\mathrm{a})}$ & $c_{2}^{(\mathrm{a})}$ \\
\hline $\mathrm{S}$ & $3 \mathrm{~s}$ & -20.00 & 1.817 & & & \\
& $3 \mathrm{p}$ & -13.30 & 1.817 & & & \\
$\mathrm{C}$ & $2 \mathrm{~s}$ & -21.40 & 1.625 & & & \\
$\mathrm{Ni}$ & $2 \mathrm{p}$ & -11.40 & 1.625 & & & \\
& $4 \mathrm{~s}$ & -9.70 & 2.10 & & & \\
& $4 \mathrm{p}$ & -5.15 & 2.10 & & & \\
& $3 \mathrm{~d}$ & -13.49 & 5.75 & 2.30 & 0.5798 & 0.5782 \\
\hline
\end{tabular}

(a) Contraction coefficients used in the double- $\zeta$ expansion. 
As reported previously [8], although the $\mathrm{Ni}(\mathrm{dmit})_{2}$ molecules play the role of acceptors in TTF[Ni(dmit $\left.)_{2}\right]_{2}$, both the highest occupied (HO) and lowest unoccupied (LU) molecular orbitals of $\mathrm{Ni}(\mathrm{dmit})_{2}$ lead to partially filled bands. This is due to the fact that the splitting between the HOMO and LUMO in the monomer - due to metal-ligand interactions - is overriden by the dispersion of energy levels produced by the monomer-monomer interactions along the $\mathrm{Ni}(\mathrm{dmit})_{2}$ stacks. Since the repeat unit cell of a $\mathrm{Ni}(\mathrm{dmit})_{2}$ slab contains four molecules, the band structure of the $\mathrm{Ni}(\mathrm{dmit})_{2}$ slab in the region near the Fermi level (see Fig. 5) contains two sets of four bands each, the upper one coming from the LUMO's and the lower one coming from the HOMO's.

It is clear from figure 5 that the density of states at the Fermi level $\left(N\left(\varepsilon_{\mathrm{F}}\right)\right)$ will contain contributions from both the HOMO $\left(N\left(\varepsilon_{\mathrm{F}}\right)_{\text {HOMO }}\right)$ and LUMO $\left(N\left(\varepsilon_{\mathrm{F}}\right)_{\text {LUMO }}\right)$ bands. In order to calculate these $N\left(\varepsilon_{\mathrm{F}}\right)$ values, we used a mesh of $125 \mathrm{k}$-points in the irreducible wedge of the rectangular Brillouin zone of the slab. The computed values were smoothed using gaussian functions with a half-width of $0.04 \mathrm{eV}$. The partial contributions of the different atoms were obtained via a Mulliken population analysis of the crystal orbitals [14].

The actual degree of charge transfer (CT) is not presently known. In consequence, we performed our study for different values of CT in between 0.5 and 1.0 electrons transferred per TTF molecule. Since the qualitative results of our study do not depend on the actual degree of charge transfer in between the above mentioned limits, we only report (Tab. III) the $N\left(\varepsilon_{\mathrm{F}}\right)_{\text {TOTAL }}, N\left(\varepsilon_{\mathrm{F}}\right)_{\text {Hомо }}$ and $N\left(\varepsilon_{\mathrm{F}}\right)_{\text {LUMO }}$ values calculated for the representative case of 0.75 electrons transferred per TTF molecule. The values in table III are in units of electrons per $\mathrm{eV}$ and per molecule. As could be expected from the nature of the HOMO and LUMO of $\mathrm{Ni}(\mathrm{dmit})_{2}$ [8], the $N\left(\varepsilon_{\mathrm{F}}\right)$ values for the four inner carbons are greater than those for the two outer ones. It is worth pointing out that although the total values associated with similar atoms (inner or outer) in the two different moieties (left or right) of the molecule are very

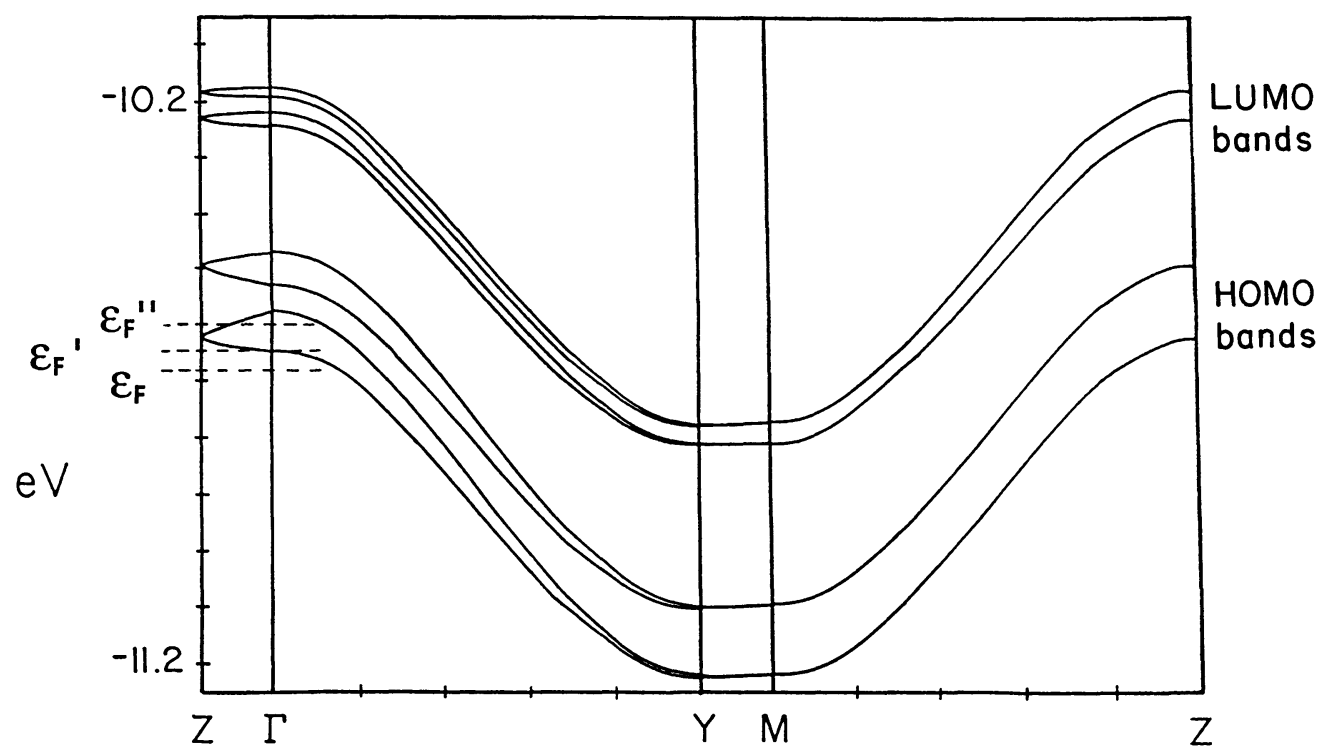

Fig. 5. - Band structure of the Ni(dmit) ${ }_{2}$ slabs in TTF[Ni(dmit $\left.)_{2}\right]_{2}$ taken from reference [8]. $\Gamma, \mathrm{Y}, \mathrm{Z}$ and $\mathrm{M}$ refer to the wavevectors $(0,0),\left(b^{*} / 2,0\right),\left(0, c^{*} / 2\right)$ and $\left(b^{*} / 2, c^{*} / 2\right)$ respectively. The Fermi levels noted $\varepsilon_{\mathrm{F}}, \varepsilon_{\mathrm{F}}^{\prime}$ and $\varepsilon_{\mathrm{F}}^{\prime \prime}$ are those appropriate for transfers of $0,0.5$ and 1.0 electrons per TTF molecule, respectively. 
Table III. - Total and partial densities of states associated with the six different carbon atoms in the slab of $\mathrm{Ni}(\mathrm{dmit})_{2}$ assuming a transfer of 0.75 electrons per TTF molecule (a).

\begin{tabular}{|l|c|c|c|}
\hline atom $^{(\mathrm{b})}$ & $N\left(\varepsilon_{\mathrm{F}}\right)_{\text {TOTAL }}{ }^{(\mathrm{c})}$ & $N\left(\varepsilon_{\mathrm{F}}\right)_{\text {HOMO }}{ }^{(\mathrm{d})}$ & $N\left(\varepsilon_{\mathrm{F}}\right)_{\mathrm{LUMO}}{ }^{(\mathrm{e})}$ \\
\hline C1 & 0.628 & 0.436 & 0.192 \\
$\mathrm{C} 2$ & 0.257 & 0.165 & 0.092 \\
$\mathrm{C} 3$ & 0.641 & 0.438 & 0.203 \\
C4 & 0.663 & 0.291 & 0.372 \\
C5 & 0.232 & 0.091 & 0.141 \\
C6 & 0.671 & 0.289 & 0.382 \\
\hline
\end{tabular}

(a) In units of electrons per $\mathrm{eV}$ and per molecule.

(b) For numbering see figure 3.

(c) Contributions associated with the eight bands of figure 5 .

(d) Contributions associated with the four lower bands of figure 5.

(e) Contributions associated with the four upper bands of figure 5.

similar, the contributions originating from the HOMO and LUMO bands are not. The possible relation between these results and the temperature dependences reported before will be discussed in the next section.

\section{Discussion.}

Figure 6 shows the locally resolved ${ }^{13} \mathrm{C}$ Knight shifts for $\mathrm{Ni}(\mathrm{dmit})_{2} \mathrm{TTF}\left[\mathrm{Ni}(\mathrm{dmit})_{2}\right]_{2}$ as a function of temperature between 160 and $295 \mathrm{~K}$. Those associated with outer carbons

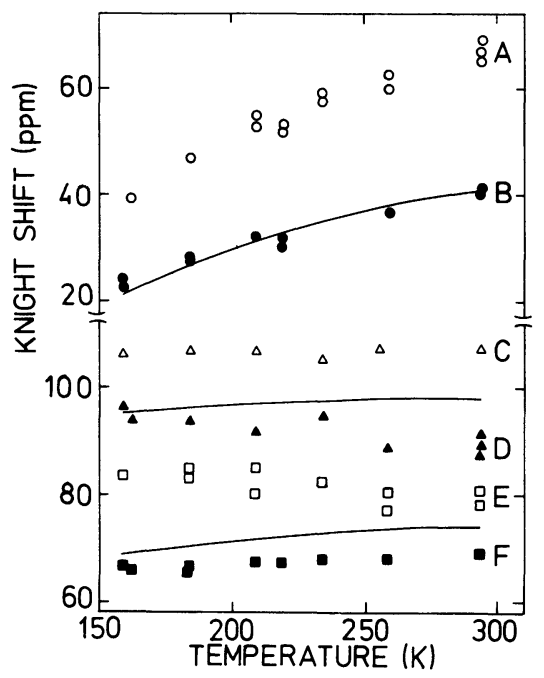

Fig. 6. $-{ }^{13} \mathrm{C}$ Knight shifts of outer $(\mathrm{A}, \mathrm{B})$ and inner (C, D, E, F) sites of $\mathrm{Ni}(\mathrm{dmit})_{2}$ versus temperature. The solid curves are fits obtained from experimental data for resonance $A$ under the assumption that the CDW instability is driven by the LUMO bands. For C-D and E-F pairs of resonances the Knight shifts averaged over the pair have been calculated (see text). 
decrease by a factor of about 1.7 towards low temperature. By contrast, the variations are small for inner carbons and do not exceed $7 \%$. To our knowledge, this is the first observation of such a different temperature behaviour of the local Knight shifts for the same molecule in an organic conductor. Temperature dependent Knight shifts have been reported for (TMTSF) $)_{2} \mathrm{PF}_{6}$ [15], (TMTSF) $)_{2} \mathrm{ReO}_{4}$ [16] and (FA) ${ }_{2} \mathrm{AsF}_{6}$ [17]. In these cases, however, they decrease at low temperatures for all carbon sites of the cation radical.

In the case of a single conduction band the Knight shift can be written as [18]

$$
K_{j}=\chi_{\mathrm{P}}\left(a_{j} / \hbar \gamma_{\mathrm{e}} \gamma_{\mathrm{n}}\right) \rho_{j}
$$

where the index $j$ denotes nonequivalent crystal sites, $\chi_{\mathrm{P}}$ is the Pauli susceptibility per one molecule, $a_{j}$ is the isotropic hyperfine interaction constant in radians per inverse second and $\rho_{j}$ is the spin density normalized to the unity over the ion-radical. $a_{j}$ and $\rho_{j}$ are mainly given by the free ion-radical parameters which are temperature independent and are slightly modified by crystal effects. So the temperature dependence of the Knight shift is expected to be of the type $K_{j}(T) \alpha \chi_{\mathrm{P}}(T)$, i.e. the same for the nuclei at different crystal sites. Our experimental data disagree with this prediction and consequently suggest that we are out of a single conduction band situation. Therefore, in the discussion which follows we use the HOMO and LUMO bands picture [8] described in the previous section.

We associate the temperature dependence of the Knight shifts in TTF[Ni(dmit) $\left.)_{2}\right]_{2}$ with the CDW instabilities known from X-ray diffuse scattering studies [5]. At room temperature and below, 1D structural fluctuations with the wave vector $0.4 b^{*}$ (the crystal $b$-axis is along the conducting chains) have been observed. They become $3 \mathrm{D}$ ordered at about $40 \mathrm{~K}$. Two additional weak 1D distortions at wave vectors $\sim 0.18 b^{*}$ and $\sim 0.22 b^{*}$ appear at $\sim 25 \mathrm{~K}$ [19]. From our previous broad band ${ }^{13} \mathrm{C}$ NMR studies the first $\mathrm{CDW}$ was proved to involve the $\mathrm{Ni}(\mathrm{dmit})_{2}$ stacks and to decrease the Knight shift averaged over the six carbon atoms $(\bar{K})$ below $100 \mathrm{~K}$ [7]. The present experiments clearly show that this decrease starts already at room temperature but the change $\Delta \bar{K} \approx 7 \mathrm{ppm}$ is too small to be detectable by broad band NMR.

Theoretical studies [20] have shown that the CDW fluctuation regime is characterized by a pseudogap in the electronic density of states which transforms into a real energy gap at the Fermi level below the temperature of the CDW 3D ordering. The temperature domain of the fluctuations is large for strongly 1D systems. In this temperature region the density of states at the Fermi level and, hence, the Knight shift decrease towards low temperature. The effect was observed up to the room temperature for ${ }^{13} \mathrm{C} \mathrm{Knight} \mathrm{shifts} \mathrm{of} \mathrm{TTF} \mathrm{[21]} \mathrm{and} \mathrm{TCNQ} \mathrm{[22]} \mathrm{in}$ TTF-TCNQ although the CDW's at the TCNQ and TTF stacks become 3D ordered at sufficiently low temperatures, $54 \mathrm{~K}$ and $38 \mathrm{~K}$, respectively.

Let us now compare the experimental Knight shifts with the calculated band structure parameters of section 3. We rewrite equation (1), as usual for a metal, as [23]

$$
K_{j}=-\mu_{\mathrm{B}} N_{j}\left(\varepsilon_{\mathrm{F}}\right) H_{j}^{\mathrm{hf}}
$$

where $H_{j}^{\mathrm{hf}}=-a_{j} / 2 \gamma_{\mathrm{n}}$ is the hyperfine field per electron, $N_{j}\left(\varepsilon_{\mathrm{F}}\right)$ is the partial contribution of the atom $j$ to the density of states at the Fermi level related to the Pauli susceptibility $\chi_{\mathrm{P}}$ by $\left|\rho_{j}\right| \chi_{\mathrm{P}}=\mu_{\mathrm{B}}^{2} N_{j}\left(\varepsilon_{\mathrm{F}}\right)$. Taking into account both the HOMO and LUMO conduction bands in TTF[Ni(dmit) $\left.)_{2}\right]_{2}$ we may write

$$
K_{j}=K_{j}^{\text {HOMO }}+K_{j}^{\text {LUMO }}
$$


where the contributions $K_{j}^{\mathrm{HOMO}}$ and $K_{j}^{\mathrm{LUMO}}$ are given by equation (2). Therefore, for the outer carbons $\mathrm{C} 2$ and $\mathrm{C} 5$ we get

$$
\begin{aligned}
& K_{\mathrm{C} 2}=-\mu_{\mathrm{B}}\left(N_{\mathrm{C} 2}^{\mathrm{H}} H_{\mathrm{o}}^{\mathrm{H}}+N_{\mathrm{C} 2}^{\mathrm{L}} H_{\mathrm{o}}^{\mathrm{L}}\right) \\
& K_{\mathrm{C} 5}=-\mu_{\mathrm{B}}\left(N_{\mathrm{C} 5}^{\mathrm{H}} H_{\mathrm{o}}^{\mathrm{H}}+N_{\mathrm{C} 5}^{\mathrm{L}} H_{\mathrm{o}}^{\mathrm{L}}\right)
\end{aligned}
$$

where $N^{\mathrm{H}}$ and $N^{\mathrm{L}}$ are the densities of states at the Fermi level for the HOMO and LUMO bands. It should be noted that the same hyperfine fields $H_{\mathrm{o}}^{\mathrm{H}}$ and $H_{\mathrm{o}}^{\mathrm{L}}$ (index « o " states the outer carbons) are supposed for both $\mathrm{C} 2$ and $\mathrm{C} 5$ sites because of their equivalence by symmetry in the free molecule. A nonequivalence of these atoms in the crystal is taken into account by their partial densities of states at the Fermi level (Tab. III). Because of this reason we also use the same hyperfine fields $H_{i}^{\mathrm{H}}$ and $H_{i}^{\mathrm{L}}$ for all the four inner carbons. We solve the system of equations (4) using the experimental Knight shifts (Tab. I) and the calculated densities of states (Tab. III) to get $H_{\mathrm{o}}^{\mathrm{H}}$ and $H_{\mathrm{o}}^{\mathrm{L}}$. Then from relation (2) we find the contributions $K^{\text {HOMO }}$ and $K^{\text {LUMO }}$. Since there are two possible assignments of the resonance lines to the $\mathrm{C} 2$ and $\mathrm{C} 5$ sites, we get two solutions as shown in table IV.

In analogy with equations (4) we may write for the inner carbon sites :

$$
\begin{aligned}
& K_{\mathrm{C} 1}+K_{\mathrm{C} 3}=-\mu_{\mathrm{B}}\left(\left(N_{\mathrm{C} 1}^{\mathrm{H}}+N_{\mathrm{C} 3}^{\mathrm{H}}\right) H_{i}^{\mathrm{H}}+\left(N_{\mathrm{C} 1}^{\mathrm{L}}+N_{\mathrm{C} 3}^{\mathrm{L}}\right) H_{i}^{\mathrm{L}}\right) \\
& K_{\mathrm{C} 4}+K_{\mathrm{C} 6}=-\mu_{\mathrm{B}}\left(\left(N_{\mathrm{C} 4}^{\mathrm{H}}+N_{\mathrm{C} 6}^{\mathrm{H}}\right) H_{i}^{\mathrm{H}}+\left(N_{\mathrm{C} 4}^{\mathrm{L}}+N_{\mathrm{C} 6}^{\mathrm{L}}\right) H_{i}^{\mathrm{L}}\right) .
\end{aligned}
$$

Here each equation describes a pair of the inner carbons of the same dmit moiety and not the individual carbons. This approach has two advantages. First, we only have to consider two possible assignments of the C-D and E-F pairs of lines to two dmit moieties of $\mathrm{Ni}(\mathrm{dmit})_{2}$ instead of eight for individual lines. Second, an analysis reveals that solutions for the pairs are much less sensitive to errors in the calculated densities of states (Tab. III) than solutions for individual sites. Thus this approach is believed to be more reliable. The calculated average over a pair of the contributions of the HOMO and LUMO bands to the Knight shifts are presented in table IV.

The results in table IV show obviously that only one set of solutions (at the right side of Tab. IV) is in agreement with the experimental temperature dependences of the Knight shifts. It corresponds to the assignments A (C5), B (C2), C-D (C1, C3) and E-F (C4, C6). The dominant contribution to the Knight shifts of the outer sites $\mathrm{C} 2$ and $\mathrm{C} 5$ arise from the LUMO bands whereas the contribution of the HOMO bands is the main one for the inner site pairs C1-C3 and C4-C6. Assuming that the CDW instability involves only the LUMO bands,

Table IV. - Contributions of the HOMO ( $\left.K^{\mathrm{HOMO}}\right)$ and the LUMO ( $\left.K^{\mathrm{LUMO}}\right)$ bands to the total Knight shift $(K)$ calculated for different assignments of the resonance lines. For $C, D$ and $E, F$ pairs of lines the averaged values over the pair are given. All shifts are in ppm.

\begin{tabular}{|l|l|l|l|l|l|r|r|}
\hline Resonance & $K$ & Nucleus & $K^{\text {HOMO }}$ & $K^{\text {LUMO }}$ & Nucleus & $K^{\text {HOMO }}$ & $K^{\text {LUMO }}$ \\
\hline A & 68 & C2 & 64.4 & 3.6 & C5 & -2.9 & 70.9 \\
B & 41 & C5 & 35.5 & 5.5 & C2 & -5.3 & 46.3 \\
C, D & 97.25 & C4, C6 & 22.4 & 74.8 & C1, C3 & 90.5 & 6.8 \\
E, F & 73 & C1, C3 & 33.8 & 39.2 & C4, C6 & 60.0 & 13.0 \\
\hline
\end{tabular}


we expect decreasing and almost constant Knight shifts for the outer and inner carbons, respectively. Using as the reference the position of the strongest temperature dependent line A and data of table IV, we can estimate the Knight shifts of the other lines versus temperature. Figure 5 shows that a satisfactory fit for line B and almost temperature independent average positions for the C-D and E-F pairs of lines are obtained. The assignement of the CDW gap to the $\mathrm{Ni}(\mathrm{dmit})_{2}$ LUMO band agrees with one of the suggestions made after comparison between the X-ray pattern and the band calculation [8].

\section{Concluding remarks.}

The main result of the present work is the observation of two different behaviours of the locally resolved ${ }^{13} \mathrm{C}$ Knight shifts for the same $\mathrm{Ni}(\mathrm{dmit})_{2}$ molecule of the TTF[Ni(dmit $\left.)_{2}\right]_{2}$ organic conductor. We observe a strong decrease towards low temperature for the outer carbons and a weak temperature dependence for the inner carbons. We believe these data provide evidence for an unusual multiband structure in organic conductors in which both the HOMO and LUMO bands of the $\mathrm{Ni}(\mathrm{dmit})_{2}$ acceptor stacks cross the Fermi level, as it was predicted by band structure calculations [8]. The drop of the outer carbon Knight shifts is associated with 1D structural fluctuations arising from the CDW instability.

Furthermore, we have tried to ascertain if the calculated contributions of the different carbon atoms to the density of states at the Fermi level are consistent with the measured distribution of their Knight shifts. Such analysis allows to test the ability of band structure calculations ignoring electron correlations to predict not only qualitative features (such as an overlap of the bands) but also more delicate details of the electronic structure. Incompleteness of the assignment of the NMR lines prevents an unambiguous answer. However, there is good agreement for one of four possible assignments. In this case the high temperature instability is driven by the LUMO bands as it was suggested for $\alpha^{\prime}-T T F\left[\operatorname{Pd}(\mathrm{dmit})_{2}\right]_{2}[8]$. The dominant contribution to the Knight shifts of the outer and inner carbons arise from the LUMO and HOMO bands, respectively. In view of these results, a ${ }^{13} \mathrm{C}$ MAS NMR study of $\left.\left(\mathrm{CH}_{3}\right)_{4} \mathrm{Ni}(\mathrm{dmit})_{2}\right)_{2}$ [24], where the conduction band is built from the LUMO [8], would be very interesting. The same type of experiments on $\alpha^{\prime}-\mathrm{TTF}\left[\operatorname{Pd}(\mathrm{dmit})_{2}\right]_{2}$ would provide an interesting comparison with the present results.

\section{Acknowledgments.}

The authors would like to thank F. Creuzet for his participation on the initial stages of this work. We also thank F. Devreux for allowing us to perform measurements on a MSL 360 NMR spectrometer and M. Smith from Bruker Laboratory at Karisruhe for his help in some experiments. The stay of A. V. at Orsay was supported by the European contract ESPRIT 3121.

\section{References}

[1] Bousseau M., Valade L., Legros J. P., Cassoux P., Garbauskas M., Interrante L. V., $J$. Am. Chem. Soc. 108 (1986) 1908.

[2] Brossard L., Ribault M., Bousseau M., Valade L., Cassoux P., C. R. Acad. Sci. Paris 302 (1986) 205.

[3] Kobayashi A., Kim H., Sasaki Y., Kato R., Kobayashi H., Moriyama S., Nishio Y., Kajita K., SASAKI W., Chem. Lett. (1987) 1819.

[4] Brossard L., Ribault M., Valade L., Cassoux P., J. Phys. France 50 (1989) 1521.

[5] Ravy S., Pouget J. P., Valade L., Legros J. P., Europhys. Lett. 9 (1989) 391. 
[6] Bourbonnais C., Wzietex P., Jérome D., Creuzet F., Valade L., Cassoux P., Europhys. Lett. 6 (1988) 177.

[7] Vainrub A., Jérome D., Bruniquel M.-F., Cassoux P., Europhys. Lett. 12 (1990) 267.

[8] Canadell E., Rachidi I. E.-I., Ravy S., Pouget J. P., Brossard L., Legros J. P., J. Phys. France 50 (1989) 2967.

[9] Mehring M., High Resolution NMR in Solids (Springer-Verlag, Berlin) 1983.

[10] Raleigh D. P., LevitT M. H., Griffin R. G., Chem. Phys. Lett. 146 (1988) 71.

[11] Hoffmann R., J. Chem. Phys. 39 (1963) 1397 ;

Whangbo M.-H., Hoffmann R., J. Am. Chem. Soc. 100 (1978) 6093.

[12] Clementi E., Roetti C., At. Nucl. Data Tables 14 (1974) 177.

[13] Ammeter J. H., Bürgi H.-B., Thibeault J., Hoffmann R., J. Am. Chem. Soc. 100 (1978) 3686.

[14] Mulliken R. S., J. Chem. Phys. 23 (1955) 1833, 2343.

[15] Stein P. C., Bernier P., Phys. Rev. B 37 (1988) 10637.

[16] Stein P. C., Bernier P., Lenoir C., Phys. Rev. B 35 (1987) 4389.

[17] Köngeter D., Mehring M., Phys. Rev. B 39 (1989) 6361.

[18] Mehring M., Spengler J., Phys. Rev. Lett. 53 (1984) 2441.

[19] Ravy S., Canadell E., Pouget J. P., The Physics and Chemistry of Organic Superconductors, Eds. G. Saito and S. Kagoshima, Proc. Phys. (Springer-Verlag) 51 (1990).

[20] Lee P. A., Rice T. M., Anderson P. W., Phys. Rev. Lett. 31 (1973) 462 ; Solid State Commun. 14 (1974) 703.

[21] Takahashi T., Jérome D., Masin F., Fabre J. M., Giral L., J. Phys. C Solid State Phys., 17 (1984) 3777.

[22] Rybaczewski E. F., Smith L. S., Garito A. F., Heeger A. J., Silbernagel B. G., Phys. Rev. $B 14$ (1976) 2746.

[23] Narath A., Hyperfine Interactions, Eds. A. J. Freeman and R. B. Frankel (Academic Press, New York) 1967.

[24] Kim H., Kobayashi A., Sasaki Y., Kato R., Kobayashi H., Chem. Lett. (1987) 1799. 